\title{
Domperidone Solubility in Aqueous Cosolvent Mixtures of $N, N$-Dimethylformamide, Isopropanol, Dimethyl Sulfoxide and Ethanol: Thermodynamic Modeling and Preferential Solvation Analysis
}

Qiong Hel, Min Zheng', Hongkun Zhao ${ }^{2, *}$

${ }^{1}$ Changzhou Vocational Institute of Engineering, School of Pharmaceutical and Environmental Engineering, Jiangsu Changzhou, 213164, People's Republic of China

${ }^{2}$ College of Chemistry \& Chemical Engineering, YangZhou University, YangZhou, Jiangsu 225002, People's Republic of China

\section{- AUTHOR INFORMATION}

Corresponding author email: hkzhao@yzu.edu.cn. 


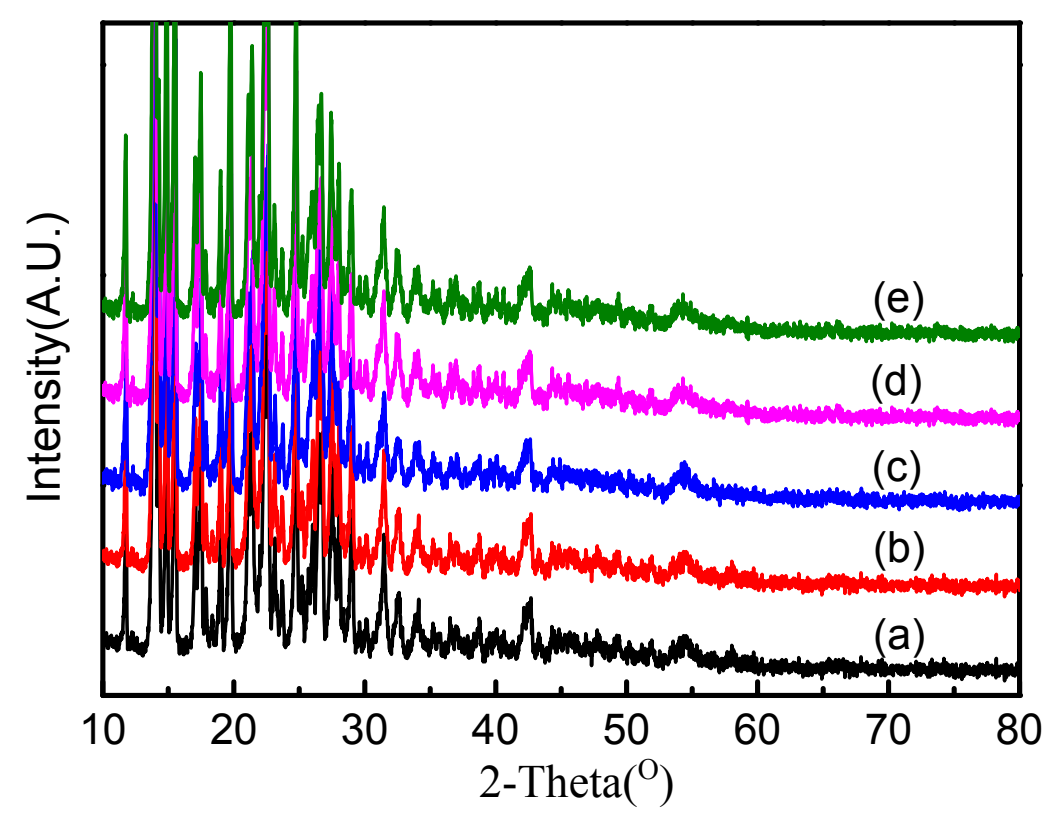

Fig. S1. XRD patterns of domperidone: a, raw domperidone; b, equilibrated with DMF + water mixture; c, equilibrated with DMSO + water mixture; d, equilibrated with isopropanol + water mixture; e, equilibrated with ethanol + water mixture. 

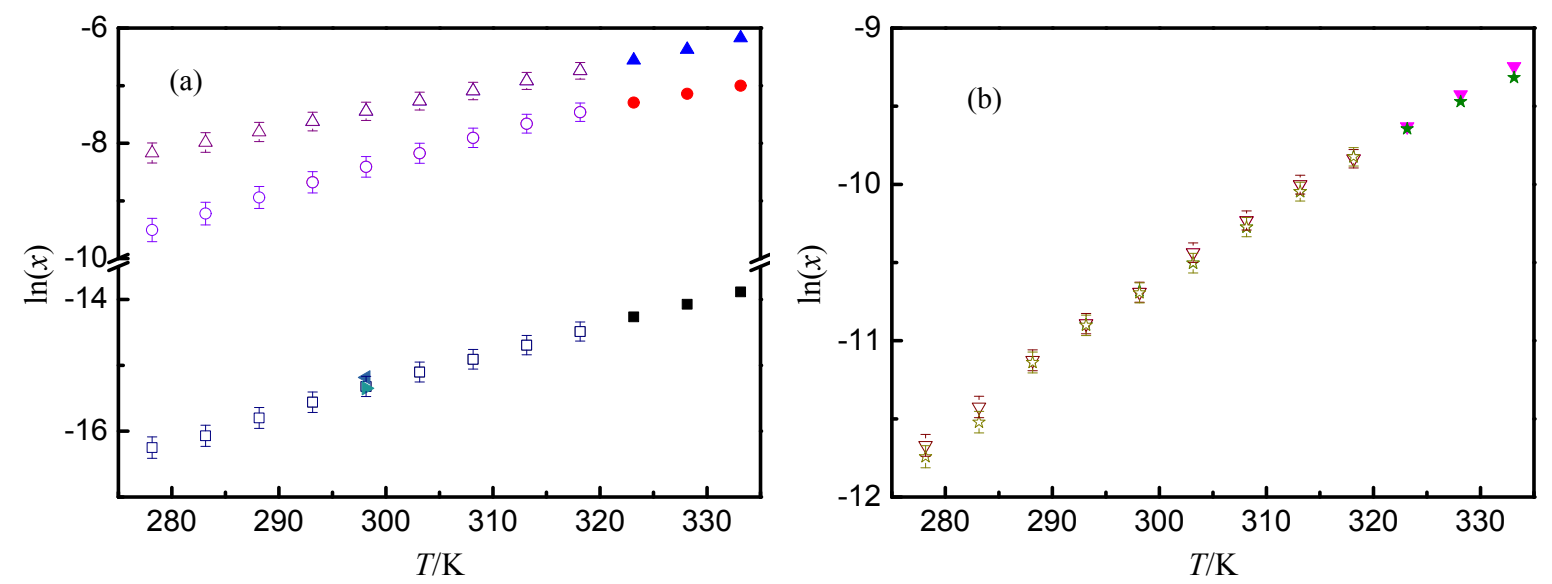

Fig. S2. Solubility of domperidone in five neat solvents determined in this work and reported in the literatures: (a):

-, water, this work; $\bullet$, DMF, this work; $\boldsymbol{\Lambda}$, DMSO, this work; $\square$, water[S1]; ○, DMF [S1]; $\triangle$, DMSO [S1]; water [S2,S3]; $\nabla$, water [S4]. (b): $\mathbf{\nabla}$, ethanol, this work; $\star$, isopropanol, this work; $\nabla$, ethanol [S1]; $\downarrow$, isopropanol [S1]. 


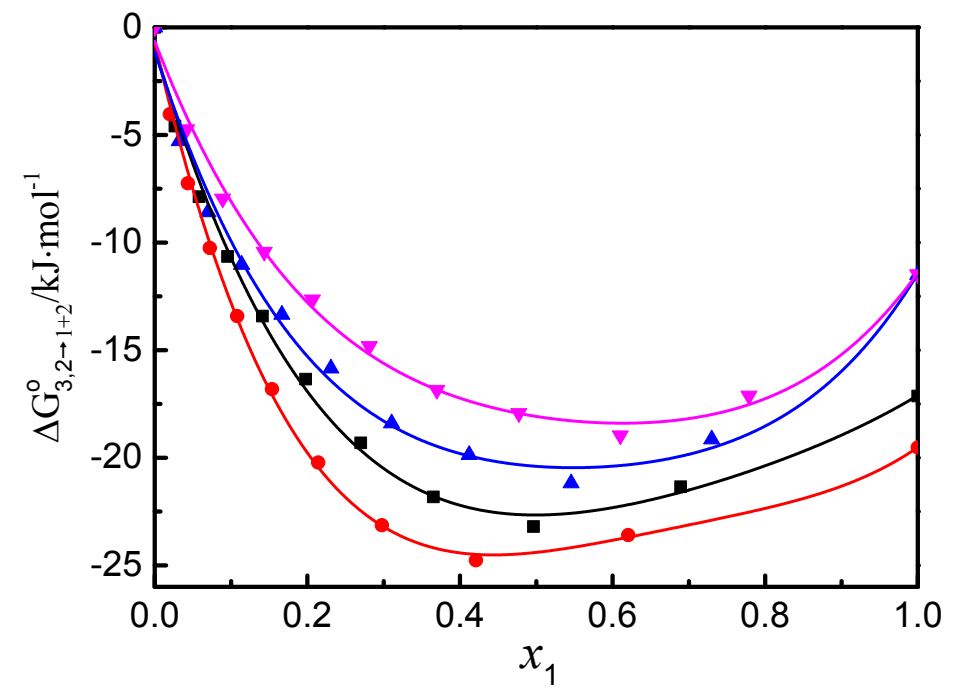

Fig. S3. Gibbs energy of transfer $\left(\mathrm{kJ} \cdot \mathrm{mol}^{-1}\right)$ of domperidone (3) from neat water (2) to DMF (1) + water (2), DMSO (1) + water (2), isopropanol (1) + water (2) and ethanol (1) + water (2) mixtures at 298.15 K. ๓, DMF (1) + water (2); $\bullet$, DMSO (1) + water (2); $\mathbf{\Lambda}$, isopropanol (1) + water (2); $\mathbf{\nabla}$, ethanol (1) + water (2). 
Table S1 Gibbs energy of transfer $\left(\mathrm{kJ} \cdot \mathrm{mol}^{-1}\right)$ of domperidone (3) from neat water (2) to DMF (1) + water (2), DMSO (1) + water (2), isopropanol (1) + water (2) and ethanol (1) + water (2) solvent mixtures at 298.15 K.

\begin{tabular}{lllll}
\hline$w_{1}{ }^{\mathrm{a}}$ & DMF (1) + water (2) & DMSO (1) + water (2) & isopropanol (1) + water (2) & ethanol (1) + water (2) \\
\hline 0 & 0 & 0 & 0 & 0 \\
0.1000 & -4.587 & -4.037 & -5.284 & -4.739 \\
0.2000 & -7.872 & -7.248 & -8.584 & -7.962 \\
0.3000 & -10.649 & -10.252 & -11.031 & -10.433 \\
0.4000 & -13.416 & -13.417 & -13.352 & -12.650 \\
0.5000 & -16.351 & -16.812 & -15.850 & -14.819 \\
0.6000 & -19.308 & -20.221 & -18.398 & -16.851 \\
0.7000 & -21.820 & -23.138 & -19.869 & -17.929 \\
0.8000 & -23.197 & -24.767 & -21.180 & -18.971 \\
0.9000 & -21.348 & -23.595 & -19.143 & -17.119 \\
1 & -17.134 & -19.524 & -11.499 & -11.480 \\
\hline
\end{tabular}

${ }^{\mathrm{a}} w$ represents the mass fraction of DMF (DMSO, isopropanol or ethanol) in mixed solvents of DMF (DMSO, isopropanol or ethanol) $(w)+$ water $(1-w)$ free of solute. 
Table S2 Coefficients of Eq. (26) (kJ.mol $\left.{ }^{-1}\right)$ applied to Gibbs energy of transfer of domperidone (3) from water (2) to DMF (1) + water (2), DMSO (1) + water (2), isopropanol (1) + water (2) and ethanol (1) + water (2) solvent mixtures at $298.15 \mathrm{~K}$.

\begin{tabular}{|c|c|c|c|c|}
\hline coefficient & $\begin{array}{l}\text { DMF (1) }+ \\
\text { water (2) }\end{array}$ & $\begin{array}{l}\text { DMSO (1) + } \\
\text { water (2) }\end{array}$ & $\begin{array}{l}\text { isopropanol (1) + } \\
\text { water (2) }\end{array}$ & $\begin{array}{l}\text { ethanol (1) + water } \\
\text { (2) }\end{array}$ \\
\hline$a$ & -0.9635 & -0.7213 & -1.11092 & -0.64696 \\
\hline$b$ & -118.580 & -152.475 & -109.854 & -91.4911 \\
\hline$c$ & 229.221 & 349.371 & 240.856 & 190.137 \\
\hline$d$ & -188.618 & -340.846 & -251.856 & -198.144 \\
\hline$e$ & 61.8332 & 125.153 & 110.521 & 88.7382 \\
\hline
\end{tabular}


Table S3 Some properties associated to preferential solvation of domperidone (3) in DMF (1) + water (2) mixtures at $298.15 \mathrm{~K}$.

\begin{tabular}{|c|c|c|c|c|c|}
\hline$x_{1}^{\mathrm{a}}$ & $\begin{array}{l}D \\
\mathrm{~kJ} \cdot \mathrm{mol}^{-1}\end{array}$ & $\begin{array}{l}G_{1,3} \\
\mathrm{~cm}^{3} \cdot \mathrm{mol}^{-1}\end{array}$ & $\begin{array}{l}G_{2,3} \\
\mathrm{~cm}^{3} \cdot \mathrm{mol}^{-1}\end{array}$ & $\begin{array}{l}V_{\text {cor }} \\
\mathrm{cm}^{3} \cdot \mathrm{mol}^{-1}\end{array}$ & $100 \delta x_{1,3}$ \\
\hline 0.00 & -118.6 & -1158 & -291.0 & 1109 & 0 \\
\hline 0.05 & -97.04 & -919.1 & -423.2 & 1133 & -3.438 \\
\hline 0.10 & -78.15 & -740.6 & -493.5 & 1212 & -3.207 \\
\hline 0.15 & -61.71 & -608.3 & -521.2 & 1310 & -1.431 \\
\hline 0.20 & -47.55 & -510.8 & -520.2 & 1403 & 0.171 \\
\hline 0.25 & -35.47 & -439.2 & -500.4 & 1485 & 1.147 \\
\hline 0.30 & -25.30 & -387.1 & -468.4 & 1557 & 1.533 \\
\hline 0.35 & -16.84 & -349.4 & -428.7 & 1623 & 1.478 \\
\hline 0.40 & -9.911 & -322.4 & -384.6 & 1683 & 1.127 \\
\hline 0.45 & -4.328 & -303.5 & -337.8 & 1741 & 0.599 \\
\hline 0.50 & 0.094 & -290.5 & -289.6 & 1797 & -0.015 \\
\hline 0.55 & 3.542 & -281.9 & -240.3 & 1852 & -0.648 \\
\hline 0.60 & 6.202 & -276.5 & -189.8 & 1907 & -1.249 \\
\hline 0.65 & 8.258 & -273.5 & -137.5 & 1962 & -1.782 \\
\hline 0.70 & 9.896 & -272.3 & -82.02 & 2018 & -2.216 \\
\hline 0.75 & 11.30 & -272.4 & -21.73 & 2075 & -2.520 \\
\hline 0.80 & 12.66 & -273.8 & 45.16 & 2134 & -2.653 \\
\hline 0.85 & 14.16 & -276.2 & 119.9 & 2194 & -2.554 \\
\hline 0.90 & 15.98 & -279.8 & 202.1 & 2258 & -2.140 \\
\hline 0.95 & 18.31 & -284.5 & 289.2 & 2325 & -1.317 \\
\hline 1.00 & 21.34 & -290.5 & 375.8 & 2398 & 0 \\
\hline
\end{tabular}

\footnotetext{
${ }^{a} x_{1}$ is the mole fraction of DMF (1) in the DMF (1) + water (2) mixtures free of domperidone (3).
} 
Table S4 Some properties associated to preferential solvation of domperidone (3) in DMSO (1) + water (2) mixtures at $298.15 \mathrm{~K}$.

\begin{tabular}{|c|c|c|c|c|c|}
\hline$x_{1}{ }^{\mathrm{a}}$ & $\begin{array}{l}D \\
\mathrm{~kJ} \cdot \mathrm{mol}^{-1}\end{array}$ & $\begin{array}{l}G_{1,3} \\
\mathrm{~cm}^{3} \cdot \mathrm{mol}^{-1}\end{array}$ & $\begin{array}{l}G_{2,3} \\
\mathrm{~cm}^{3} \cdot \mathrm{mol}^{-1}\end{array}$ & $\begin{array}{l}V_{\text {cor }} \\
\mathrm{cm}^{3} \cdot \mathrm{mol}^{-1}\end{array}$ & $100 \delta x_{1,3}$ \\
\hline 0.00 & -152.5 & -1407 & -291.0 & 1110 & 0 \\
\hline 0.05 & -120.0 & -1155 & -458.5 & 1103 & -5.427 \\
\hline 0.10 & -92.33 & -909.0 & -547.4 & 1170 & -5.553 \\
\hline 0.15 & -68.98 & -700.6 & -564.9 & 1276 & -2.503 \\
\hline 0.20 & -49.62 & -543.8 & -534.1 & 1370 & -0.185 \\
\hline 0.25 & -33.88 & -436.0 & -479.8 & 1445 & 0.842 \\
\hline 0.30 & -21.36 & -367.0 & -420.3 & 1509 & 1.013 \\
\hline 0.35 & -11.71 & -325.5 & -365.9 & 1567 & 0.757 \\
\hline 0.40 & -4.545 & -302.0 & -321.3 & 1622 & 0.353 \\
\hline 0.45 & 0.513 & -289.9 & -287.3 & 1676 & -0.045 \\
\hline 0.50 & 3.838 & -284.4 & -263.4 & 1731 & -0.360 \\
\hline 0.55 & 5.805 & -282.6 & -247.6 & 1787 & -0.570 \\
\hline 0.60 & 6.789 & -282.7 & -237.9 & 1843 & -0.682 \\
\hline 0.65 & 7.166 & -283.6 & -231.7 & 1899 & -0.723 \\
\hline 0.70 & 7.311 & -284.6 & -226.2 & 1955 & -0.727 \\
\hline 0.75 & 7.600 & -285.3 & -217.6 & 2010 & -0.730 \\
\hline 0.80 & 8.408 & -285.8 & -200.4 & 2064 & -0.761 \\
\hline 0.85 & 10.11 & -285.9 & -165.7 & 2118 & -0.829 \\
\hline 0.90 & 13.08 & -286.0 & -95.72 & 2170 & -0.900 \\
\hline 0.95 & 17.70 & -286.8 & 51.32 & 2224 & -0.822 \\
\hline 1.00 & 24.34 & -290.8 & 409.0 & 2285 & 0 \\
\hline
\end{tabular}

\footnotetext{
${ }^{a} x_{1}$ is the mole fraction of DMSO (1) in the DMSO (1) + water (2) mixtures free of domperidone (3).
} 
Table S5 Some properties associated to preferential solvation of domperidone (3) in isopropanol (1) + water (2) mixtures at $298.15 \mathrm{~K}$.

\begin{tabular}{|c|c|c|c|c|c|}
\hline$x_{1}^{\mathrm{a}}$ & $\begin{array}{l}D \\
\mathrm{~kJ} \cdot \mathrm{mol}^{-1}\end{array}$ & $\begin{array}{l}G_{1,3} \\
\mathrm{~cm}^{3} \cdot \mathrm{mol}^{-1}\end{array}$ & $\begin{array}{l}G_{2,3} \\
\mathrm{~cm}^{3} \cdot \mathrm{mol}^{-1}\end{array}$ & $\begin{array}{l}V_{\text {cor }} \\
\mathrm{cm}^{3} \cdot \mathrm{mol}^{-1}\end{array}$ & $100 \delta x_{1,3}$ \\
\hline 0.00 & -109.9 & -1091 & -291.0 & 1107 & 0 \\
\hline 0.05 & -87.60 & -916.0 & -389.5 & 1122 & -3.541 \\
\hline 0.10 & -68.80 & -783.6 & -456.5 & 1164 & -4.362 \\
\hline 0.15 & -53.11 & -681.3 & -501.6 & 1225 & -3.292 \\
\hline 0.20 & -40.20 & -600.7 & -530.6 & 1291 & -1.504 \\
\hline 0.25 & -29.74 & -536.5 & -547.6 & 1356 & 0.257 \\
\hline 0.30 & -21.41 & -484.7 & -555.1 & 1416 & 1.676 \\
\hline 0.35 & -14.86 & -441.8 & -553.6 & 1472 & 2.656 \\
\hline 0.40 & -9.767 & -405.0 & -541.2 & 1523 & 3.155 \\
\hline 0.45 & -5.801 & -370.4 & -509.6 & 1568 & 3.071 \\
\hline 0.50 & -2.630 & -333.4 & -437.9 & 1606 & 2.140 \\
\hline 0.55 & 0.080 & -288.2 & -283.2 & 1631 & -0.092 \\
\hline 0.60 & 2.659 & -234.7 & 2.385 & 1643 & -3.787 \\
\hline 0.65 & 5.439 & -190.6 & 377.2 & 1651 & -7.784 \\
\hline 0.70 & 8.751 & -177.9 & 678.8 & 1674 & -10.263 \\
\hline 0.75 & 12.93 & -191.5 & 833.0 & 1714 & -10.798 \\
\hline 0.80 & 18.30 & -213.8 & 898.3 & 1767 & -10.022 \\
\hline 0.85 & 25.20 & -235.9 & 936.5 & 1827 & -8.462 \\
\hline 0.90 & 33.96 & -255.6 & 976.1 & 1892 & -6.301 \\
\hline 0.95 & 44.90 & -273.0 & 1026 & 1962 & -3.519 \\
\hline 1.00 & 58.37 & -288.8 & 1089 & 2039 & 0 \\
\hline
\end{tabular}

\footnotetext{
${ }^{\mathrm{a}} x_{1}$ is the mole fraction of isopropanol (1) in the isopropanol (1) + water (2) mixtures free of domperidone (3).
} 
Table S6 Some properties associated to preferential solvation of domperidone (3) in ethanol (1) + water (2) mixtures at $298.15 \mathrm{~K}$.

\begin{tabular}{|c|c|c|c|c|c|}
\hline$x_{1}^{\mathrm{a}}$ & $\begin{array}{l}D \\
\mathrm{~kJ} \cdot \mathrm{mol}^{-1}\end{array}$ & $\begin{array}{l}G_{1,3} \\
\mathrm{~cm}^{3} \cdot \mathrm{mol}^{-1}\end{array}$ & $\begin{array}{l}G_{2,3} \\
\mathrm{~cm}^{3} \cdot \mathrm{mol}^{-1}\end{array}$ & $\begin{array}{l}V_{\text {cor }} \\
\mathrm{cm}^{3} \cdot \mathrm{mol}^{-1}\end{array}$ & $100 \delta x_{1,3}$ \\
\hline 0.00 & -91.49 & -957.4 & -291.0 & 1107 & 0 \\
\hline 0.05 & -73.92 & -861.4 & -380.9 & 1126 & -3.166 \\
\hline 0.10 & -59.05 & -760.3 & -448.7 & 1167 & -4.081 \\
\hline 0.15 & -46.63 & -663.3 & -491.9 & 1227 & -3.083 \\
\hline 0.20 & -36.37 & -577.4 & -512.6 & 1292 & -1.353 \\
\hline 0.25 & -28.03 & -505.9 & -515.7 & 1355 & 0.216 \\
\hline 0.30 & -21.32 & -449.2 & -506.7 & 1413 & 1.308 \\
\hline 0.35 & -15.99 & -405.5 & -490.3 & 1465 & 1.922 \\
\hline 0.40 & -11.77 & -372.4 & -469.7 & 1514 & 2.157 \\
\hline 0.45 & -8.395 & -347.1 & -445.5 & 1559 & 2.103 \\
\hline 0.50 & -5.593 & -327.1 & -415.9 & 1603 & 1.803 \\
\hline 0.55 & -3.101 & -310.0 & -374.9 & 1643 & 1.231 \\
\hline 0.60 & -0.652 & -294.0 & -311.6 & 1680 & 0.307 \\
\hline 0.65 & 2.018 & -277.9 & -209.2 & 1713 & -1.071 \\
\hline 0.70 & 5.178 & -262.0 & -49.24 & 1742 & -2.895 \\
\hline 0.75 & 9.092 & -249.4 & 172.2 & 1769 & -4.864 \\
\hline 0.80 & 14.03 & -244.3 & 423.3 & 1801 & -6.320 \\
\hline 0.85 & 20.25 & -249.0 & 643.7 & 1844 & -6.583 \\
\hline 0.90 & 28.03 & -260.9 & 789.0 & 1900 & -5.419 \\
\hline 0.95 & 37.62 & -275.5 & 858.1 & 1966 & -3.082 \\
\hline 1.00 & 49.30 & -957.4 & 874.6 & 2039 & 0 \\
\hline
\end{tabular}

${ }^{\mathrm{a}} x_{1}$ is the mole fraction of ethanol (1) in the ethanol (1) + water (2) mixtures free of domperidone (3). 


\section{- REFERENCES}

(S1) Zheng, M.; Chen, J.; Chen, G. Q.; Farajtabar, A.; Zhao, H. K. Solubility Modelling and Solvent Effect for Domperidone in Twelve Green Solvents. J. Mol. Liq. 2018, 261, 50-56.

(S2) Dave, R. A.; Morris, M. E. Novel High/Low Solubility Classification Methods for New Molecular Entities. Int. J. Pharm. 2016, 511, 111-126.

(S3) Benet, L. Z.; Broccatelli, F.; Oprea, T. I. BDDCS Applied to Over 900 Drugs. AAPS J. 2011, $13,519-547$.

(S4) Aboutaleb, A. E.; Abdel-Rahman, S. I.; Ahmed, M. O.; Younis, M. A. Improvement of Domperidone Solubility and Dissolution Rate by Dispersion in Various Hydrophilic Carriers. J. Appl. Pharm. Sci. 2016, 6, 133-139. 